\title{
Modified Kudo classification can improve accuracy of virtual chromoendoscopy with FICE in endoscopic surveillance of ulcerative colitis
}

\section{(ㄷ)(1) $(-)$}

\author{
Authors \\ Institutions \\ 1 Gastroenterology Unit, ASST Fatebenefratelli Sacco, \\ Milan, Italy \\ 2 ASST Sette Laghi, Varese (Italy) \\ 3 Pathology Unit, ASST Fatebenefratelli Sacco, Milan, Italy \\ 4 Chair of Statistics, University of Milan, Milan, Italy \\ 5 Pharmacy Service - Fatebenefratelli e Oftalmico \\ Hospital, Milan, Italy \\ 6 IBD Surgery Unit, ASST Fatebenefratelli Sacco, Milan, \\ Italy \\ 7 Endoscopy Unit, ASST Cremona, Cremona, Italy \\ 8 Intensive Care Unit, ASST Fatebenefratelli Sacco, Milan, \\ Italy
}

Andrea Cassinotti ${ }^{1,2}$, Paolo Fociani ${ }^{3}$, Piergiorgio Duca ${ }^{4}$, Manuela Nebuloni ${ }^{3}$, Sophia Elizabeth Campbell Davies ${ }^{5}$, Gianluca Sampietro ${ }^{6}$, Federico Buffoli ${ }^{7}$, Alberto Corona ${ }^{8}$, Giovanni Maconi ${ }^{1}$, Sandro Ardizzone ${ }^{1}$

submitted 5.9.2019

accepted after revision 6.4 .2020

\section{Bibliography}

Endoscopy International Open 2020; 08: E1414-E1422

DOI 10.1055/a-1165-0169

ISSN 2364-3722

(C) 2020. The Author(s).

This is an open access article published by Thieme under the terms of the Creative Commons Attribution-NonDerivative-NonCommercial License, permitting copying and reproduction so long as the original work is given appropriate credit. Contents may not be used for commecial purposes, or adapted, remixed, transformed or built upon. (https://creativecommons.org/licenses/by-nc-nd/4.0/)

\section{Corresponding author}

Andrea Cassinotti, ASST dei Sette Laghi - Gastroenterology Unit, Ospedale di Circolo e Fondazione Macchi, via Borri 57

Varese 21100, Italy

Fax: +00390239042232

andrea.cassinotti@asst-settelaghi.it
ABSTRACT

Background and study aims Virtual chromoendoscopy with Fuji Intelligent Color Enhancement (FICE) has never been studied in prospective trials of endoscopic surveillance for ulcerative colitis (UC). We compared FICE and white light endoscopy (WLE) in differentiation of visible lesions in UC.

Patients and methods In a prospective parallel study, we compared consecutive outpatients with UC submitted to surveillance colonoscopy with FICE or WLE. At least one visible polypoid or non-polypoid lesion for each patient was required. Random biopsies from normal mucosa, targeted biopsies or removal of suspected neoplastic lesions and targeted biopsies of unsuspected lesions were performed. In the FICE arm, neoplasia was suspected according to a modified Kudo classification (FICE-KUDO/inflammatory bowel disease [IBD]). Sensitivity (SE), specificity (SP), positive and negative likelihood ratios (LR) and negative predictive value (NPV) were analyzed.

Results One hundred patients were submitted to FICE ( $\mathrm{n}=$ $46)$ or WLE $(n=54)$. Twenty-two patients (11 in WLE, 11 in FICE) had a least one neoplastic lesion. No neoplasia was found in random biopsies. Among 275 lesions, 17 of 136 by FICE and 27 of 139 by WLE were suspected neoplasia, but 28 (14 in each arm) were true neoplastic lesions. The accuracy of FICE-KUDO/IBD vs WLE (per lesion) was: SE $93 \%$ vs $64 \%$ $(P=0.065)$, SP $97 \%$ vs $86 \%(P=0.002)$, positive-LR 28.3 vs $4.5(P=0.001)$, negative-LR 0.07 vs $0.42(P=0.092)$, NPV $99 \%$ vs $96 \%(P=0.083)$. FICE-KUDO/IBD detected more non-polypoid lesions than WLE $(P=0.016)$.

Conclusions Targeted biopsies of polypoid and non-polypoid lesions, using the modified Kudo classification with FICE are more accurate than WLE in UC surveillance.

\section{Introduction}

Patients with long-standing ulcerative colitis (UC) have an increased risk of colorectal cancer (CRC), which can develop through the dysplasia-carcinoma sequence. Therefore, periodic colonoscopies are performed to reduce CRC-related morbidity and mortality by detecting and removing neoplasia at the earliest stage [1].

Neoplastic lesions of colorectal mucosa in UC were formerly described as flat (endoscopically invisible) or elevated (endoscopically visible). More recently, gross morphology of neoplas- 
tic lesions in the colon and rectum has been better described as polypoid and non-polypoid, according to the Paris classification [2], but it has been used in very few studies in inflammatory bowel disease (IBD). On the other hand, similar macroscopic aspects can be seen in inflammatory, non-neoplastic, lesions, which can be even more frequent in IBD and often have been excluded from previous studies assessing diagnostic accuracy of surveillance endoscopy in UC [3].

Early international guidelines for colonoscopic surveillance in IBD used white light endoscopy (WLE) and recommended performing targeted biopsies of any visible suspected neoplastic lesion as well as multiple random biopsies along the colorectal mucosa to detect those neoplastic lesions arising in otherwise apparently normal mucosa, which were more difficult to see with standard-resolution endoscopes [1].

Recent availability of powered endoscopy, such as high-definition equipment and dye-based chromoendoscopy, has improved detection of neoplastic lesions in IBD, including the flat and non-polypoid forms [4,5]. Moreover, dye-based chromoendoscopy, when added to magnification endoscopy to better define the mucosal crypt architecture according to the pit-pattern classification of Kudo [6], has been used to differentiate neoplastic and non-neoplastic lesions, although the accuracy of this classification in the specific setting of IBD is still controversial due to inflammatory and regenerative artifacts [3].

International guidelines suggest methylene blue or indigo carmine chromoendoscopy as the preferred method for surveillance colonoscopy $[1,7]$. On the other hand, so-called "virtual" chromoendoscopy, which includes narrow band imaging (NBI, Olympus), i-SCAN (Pentax), Fuji Intelligent Color Enhancement
(FICE, Fujifilm) and Blue-laser imaging (BLI, Fujifilm), has only recently been included for routine use in the latest European Society of Gastrointestinal Endoscopy guidelines [8], despite the paucity of ad hoc controlled studies in IBD [9] and the failure of early controlled studies with unmagnified NBI compared to standard WLE or dye-based chromoendoscopy [8].

Recently, we published the first experience with FICE in IBD, which focused only on visible polypoid and non-polypoid lesions found during surveillance in UC [3]. Through a post-hoc, retrospective analysis of endoscopic predictors of neoplasia, that study showed the need to develop and validate a more accurate endoscopic classification system for characterization of raised lesions in IBD, based not only on pit-pattern visualization but also on inflammatory markers. This new classification, specific for FICE in IBD, was prospectively used for the first time in this parallel study, in which FICE was compared to WLE for differentiation of all visible lesions found in consecutive patients with UC scheduled for surveillance colonoscopy.

\section{Patients and methods}

\section{Study design and patients}

This was a prospective, observational, parallel study in which consecutive outpatients with long-lasting UC who were scheduled for surveillance colonoscopy at our center, were submitted to a withdrawal colonoscopy with WLE or FICE ( $\triangleright$ Fig. 1), depending on the availability of study instruments (only one FICE was available) at the time of the scheduled endoscopy.

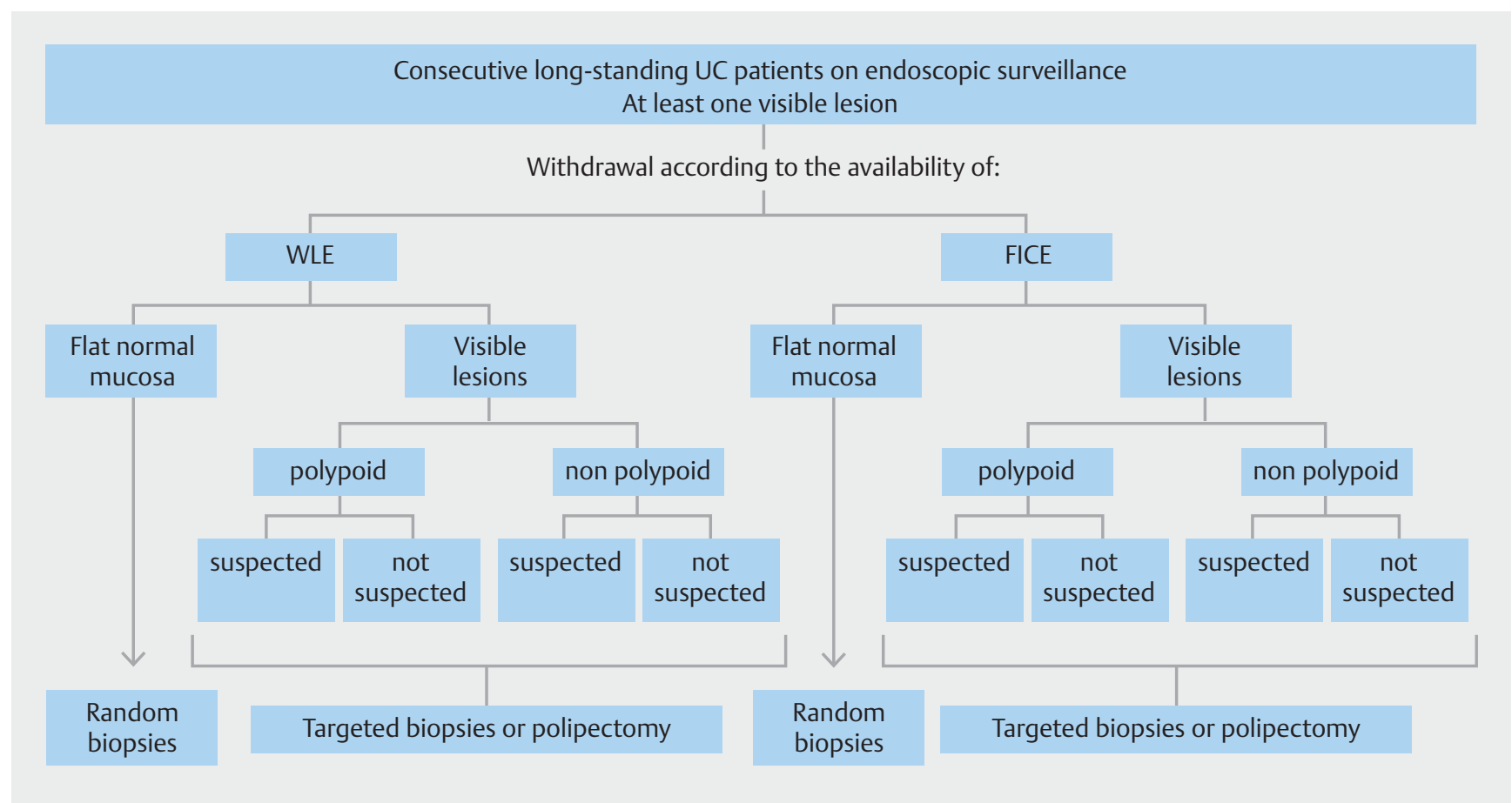

- Fig. 1 Study design and allocation of patients according to the endoscopic method and the random versus targeted protocol of sample collection. 
The study was approved by our Ethical Committee and patients signed an informed consent form to undergo endoscopic examination.

Inclusion criteria were a previous confirmed diagnosis of UC according to clinical, endoscopic, and histological data; disease duration of at least 8 years since onset of symptoms; no or mild clinical activity according to a Mayo score $\leq 4$; and presence of at least one visible polypoid or non-polypoid lesion during the surveillance colonoscopy, according to the Paris classification [2].

Exclusion criteria were proctitis, coagulopathy, pregnancy, melanosis coli, previous colorectal resection, previous colonoscopy in the last 3 months with unresected neoplasia, massive pseudopolyposis (> 30 polyps), poor bowel preparation (Boston Bowel Preparation Score $<2$ in any segment), and use of dyebased chromoendoscopy or virtual chromoendoscopy other than FICE.

The following demographic and clinical data were collected at baseline: sex, date of birth, disease onset, disease diagnosis, disease extent, personal/familiar history of colorectal neoplasia, clinical activity according to the Mayo score, extra-intestinal manifestations including primary sclerosing cholangitis (PSC), and previous and concomitant medications.

According to the parallel design of our study, patient enrolment stopped after two comparable groups of patients were obtained according to all significant demographic, clinical, and endoscopic variables, including the rate of neoplastic lesions, in order to decrease the risk of selection and detection bias in a study focused on differential diagnosis rather than detection rate of neoplasia.

\section{Endoscopic protocol}

In the WLE arm, standard white light colonoscopy was performed using the Olympus CV-180 Evis Exera II system (Olympus Corp., Tokyo, Japan). At the time of study conception (2012), this was our standard of care for endoscopic surveillance of IBD, with random biopsies performed every $10 \mathrm{~cm}$ plus targeted biopsies of suspected neoplastic lesions. FICE was then acquired by our endoscopic unit and tested in the context of a plan to validate its use in IBD.

In the FICE arm, virtual chromoendoscopy was performed with the high-definition, magnified, Fujinon EG-590ZW colonoscope (Fujinon Corp, Saitama, Japan), equipped with the EPX4400 processor. The FICE set number 4 was used exclusively, according to previous validation studies $[3,10]$. Magnification was activated according to the endoscopist's need, based on his confidence to assess the mucosal and vascular patterns of each lesion in supporting his differential diagnosis.

In both arms, assessment of the colon to search for visible lesions was performed systematically during withdrawal of the instrument with a minimum diagnostic extubation time of 10 minutes.

\section{Classification of lesions}

Morphology of each lesion was determined in accordance with the Paris classification of macroscopic aspects as polypoid or non-polypoid [2].
In the WLE arm, areas of suspected neoplasia were defined as any mucosal irregularity, ulceration, polypoid or non-polypoid lesion that was not entirely consistent with chronic or active UC according to the usual clinical practice [11].

In the FICE arm, neoplasia was first suspected according to the conventional Kudo classification (FICE-KUDO), and then according to the modified Kudo classification for FICE in IBD (FICE-KUDO/IBD) which was developed in our previous pilot study adding three specific endoscopic modifiers for risk of neoplasia [3]. In particular, as shown in > Fig. 2, Kudo pit-patterns I and II, which are usually associated with non-neoplastic lesions in non-IBD studies, were considered neoplastic lesions if associated with the presence of at least one of two further endoscopic factors:

(1) irregular brown/bluish visible capillary vessels, defined according to a Teixeira III-V vascular pattern, which were previously described in studies with FICE in the general population and were related to higher risk of neoplasia $[3,12]$; and (2) pits heterogeneity, defined as variable density or size of pits in the context of low-risk (type I and II) patterns, which was previously associated with higher risk of neoplasia $[3,13,14]$.

In contrast, Kudo pit-patterns III-IV, usually related to neoplasia in non-IBD studies [6], were considered non-neoplastic lesions if associated with a fibrin cap, defined as a non-removable, circumscribed, white exudate covering at least $25 \%$ of the surface of the lesions, which has been associated with inflammatory non-neoplastic lesions in previous studies in IBD $[3,15,16]$.

Kudo V and III-s pit-patterns, usually associated with higher risk of advanced lesions, including invasive cancer, were always considered suspicious for neoplasia, independent of presence of the three endoscopic modifiers.

Finally, lesions not classified with enough confidence by the endoscopist according to the conventional Kudo classification (which accounted up to $15 \%$ of visible lesions in our previous study [3]) were considered neoplastic, except in the presence of fibrin cap, which have been associated with non-neoplastic inflammatory lesions.

\section{Biopsy protocol}

In both arms, three types of histological samples were obtained: 1) every $10 \mathrm{~cm}$, random biopsies of otherwise normal flat mucosa, from the cecum to the rectum; 2) targeted biopsies or full endoscopic resection of visible suspected neoplastic lesions (polypoid or non-polypoid), as appropriate; and 3) targeted biopsies of unsuspected neoplastic lesions (polypoid or nonpolypoid).

Neoplastic changes were classified according to the new Vienna classification as low-grade intramucosal, high-grade intramucosal, or invasive neoplasia [17].

Non-neoplastic lesions, including hyperplasic and inflammatory polyps, were described according to current classifications.

\section{Statistical methods}

At the time this study was conceived (2012), we did not find studies comparing FICE with WLE or other types of chromoendoscopy in IBD, which precluded rigorous sample size calculation. Moreover, previous controlled studies with other chromo- 


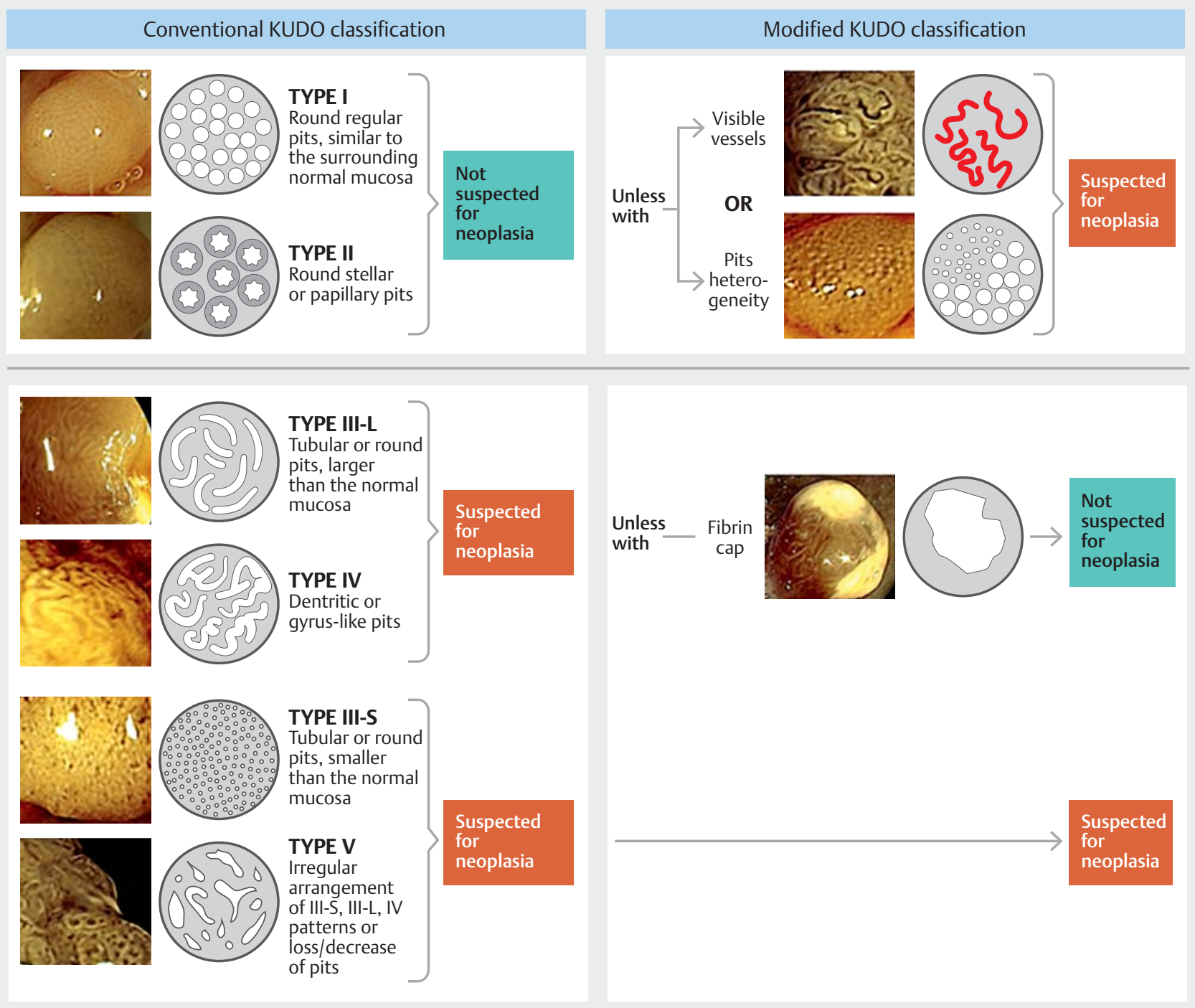

- Fig. 2 Schematic classification of lesions suspected and not suspected for neoplasia according to the modified Kudo classification, based on type and heterogeneity of pit-patterns and presence of a fibrin cap or visible microvessels (see text).

endoscopy techniques focused on dysplasia detection rather than differential diagnosis of visible lesions [4]. Therefore, according to our parallel study design, we decided a priori to collect at least 100 visible lesions in each arm, stopping the enrollment when all significant demographic, clinical, and endoscopic variables between the two groups were comparable; this included, by the way, a not significantly different rate of neoplastic visible lesions.

The diagnostic performance of FICE (according to the two classifications) and WLE was evaluated by comparing the endoscopic evaluation (suspected or unsuspected neoplasia) with the histological diagnosis (reference test).

The analyses were primarily performed per lesion, since sample size and enrollment of patients were based on the number of visible lesions. A per patient analysis was also included to permit the reader to compare our results to those from previous studies that used the same approach.
Sensitivity and specificity $95 \%$ confidence intervals (Cis) were estimated by applying the binomial exact method. Positive and negative likelihood ratio (LR) and positive (PPVs) and negative predictive values (NPVs) were also calculated. For NPV, the Preservation and Incorporation of Valuable Endoscopic Innovations (PIVI) threshold set at $90 \%$ or higher was used for adopting real-time endoscopic assessment of histology, although our sample had no limitations on polyp size [18].

Data were described using means and standard deviation, or medians and interquartile range, when appropriate. To test the difference between WLE and FICE, the $z$ test was applied and the two tails probability reported.

Statistical analyses were conducted using IBM-SPSS for Windows 24th version and Excel 2013. 


\section{Results}

\section{Patients and lesions}

Between September 2012 and October 2014, 329 patients with UC underwent surveillance colonoscopy for long-standing UC. Eighty-four patients were excluded according to various exclusion criteria ( Fig. 3 ). Among the remaining 245 patients, 145 had no visible lesions while 100 patients had at least one visible lesion (total number of lesions 275, mean number per patient 2.7) during their colonoscopy with WLE ( $n=54 ; 139$ lesions) or FICE ( $n=46 ; 136$ lesions).

Both groups of patients had comparable baseline characteristics ( Table 1 ), including similar percentages of neoplastic lesions ( $n=14$ in both arms, $10 \%$ ) and patients with neoplasia ( $n=11$ in both arms; $20 \%$ vs $24 \%$ ), as required by our study design.

All neoplastic lesions had low-grade dysplasia, except for one adenocarcinoma in the FICE group. Non-neoplastic lesions were inflammatory lesions except for 26 hyperplasic lesions (FICE $n=18$, WLE $n=8$ ).

Two-hundred and sixty-six lesions were polypoid (WLE $n=$ 138, FICE $n=128$ ) and nine were non-polypoid (WLE $n=1$, FICE $\mathrm{n}=8 ; P=0.016)$. All non-polypoid lesions in the FICE group were type Ila, while the only non-polypoid lesion in the WLE arm (non-neoplastic) was type IIb.

\section{Frequency of neoplasia in random biopsies of flat mucosa}

In the WLE arm, 1011 random biopsies were taken (mean per

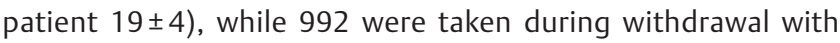

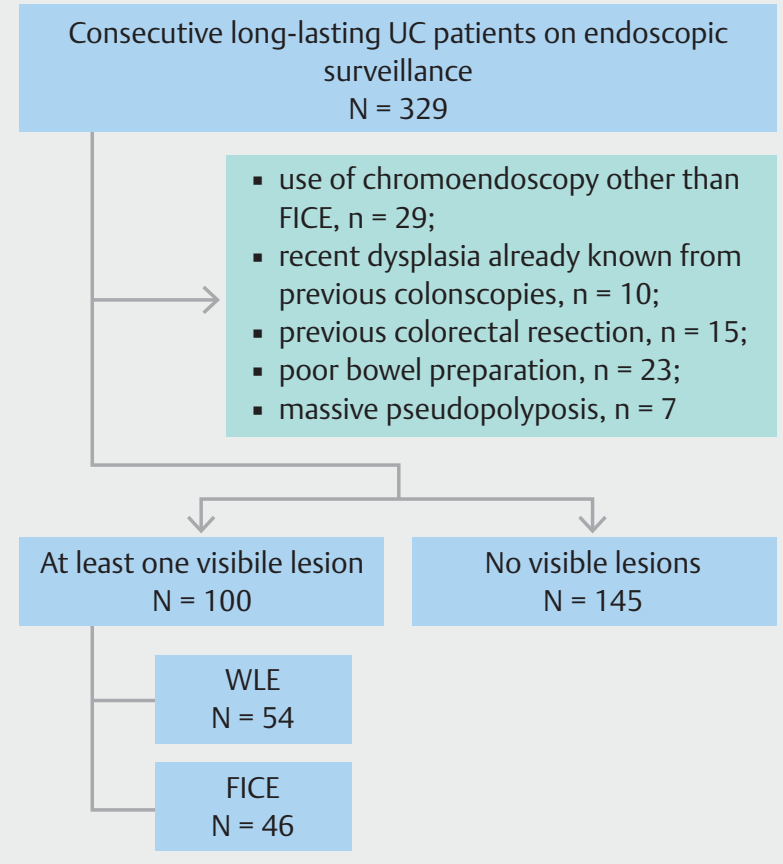

- Fig. 3 Study population overview.
- Table 1 Characteristics of patients included in the two groups of surveillance endoscopy.

\begin{tabular}{|c|c|c|}
\hline & $\begin{array}{l}\text { WLE } \\
\text { (54 patients, } \\
139 \text { lesions) }\end{array}$ & $\begin{array}{l}\text { FICE } \\
\text { (46 patients, } \\
136 \text { lesions) }\end{array}$ \\
\hline Age - mean (range), years & $56(26-84)$ & $54(34-73)$ \\
\hline Male/female - ratio & $42 / 12$ & $32 / 14$ \\
\hline $\begin{array}{l}\text { Disease duration - mean (range), } \\
\text { years }\end{array}$ & $19(8-36)$ & $19(8-44)$ \\
\hline $\begin{array}{l}\text { Extensive colitis versus left colitis } \\
\text { - rate }\end{array}$ & $67 \%$ & $78 \%$ \\
\hline $\begin{array}{l}\text { Endoscopic activity (Mayo } \geq 1 \text { ) - } \\
\text { rate }\end{array}$ & $54 \%$ & $61 \%$ \\
\hline - Mayo 1 & $17 \%$ & $26 \%$ \\
\hline - Mayo 2 & $22 \%$ & $20 \%$ \\
\hline - Mayo 3 & $15 \%$ & $15 \%$ \\
\hline Familiarity for CRC - rate & $17 \%$ & $18 \%$ \\
\hline $\begin{array}{l}\text { Primary sclerosing cholangitis - } \\
\text { rate }\end{array}$ & $0 \%$ & $0 \%$ \\
\hline \multicolumn{3}{|l|}{ Site of lesions - number (rate) } \\
\hline - Cecum & $11(8 \%)$ & $11(8 \%)$ \\
\hline - Ascending colon & $12(9 \%)$ & $22(16 \%)$ \\
\hline - Transverse colon & $23(16 \%)$ & $24(18 \%)$ \\
\hline - Descending colon & $35(25 \%)$ & $22(16 \%)$ \\
\hline - Sigmoid colon & $46(33 \%)$ & $48(35 \%)$ \\
\hline - Rectum & $12(9 \%)$ & $9(7 \%)$ \\
\hline $\begin{array}{l}\text { Size of lesions - median (range), } \\
\text { mm }\end{array}$ & $5(2-20)$ & $5(2-30)$ \\
\hline $\begin{array}{l}\text { Neoplastic lesions - number } \\
\text { (rate) }\end{array}$ & $14(10 \%)$ & $14(10 \%)$ \\
\hline \multicolumn{3}{|c|}{ Site of neoplastic lesions - number (rate) } \\
\hline - Cecum & $4(29 \%)$ & $2(14 \%)$ \\
\hline - Ascending colon & $2(14 \%)$ & $2(14 \%)$ \\
\hline - Transverse colon & $1(7 \%)$ & $0(0 \%)$ \\
\hline - Descending colon & $3(21 \%)$ & $7(50 \%)$ \\
\hline - Sigmoid colon & $3(21 \%)$ & $2(14 \%)$ \\
\hline - Rectum & $1(7 \%)$ & $1(7 \%)$ \\
\hline $\begin{array}{l}\text { Size of neoplastic lesions - medi- } \\
\text { an (range), mm }\end{array}$ & $5(3-12)$ & $7(3-30)$ \\
\hline $\begin{array}{l}\text { Inflammatory lesions - number } \\
\text { (rate) }\end{array}$ & $117(84 \%)$ & $104(76 \%)$ \\
\hline $\begin{array}{l}\text { Patients with neoplasia - number } \\
\text { (rate) }\end{array}$ & $11(20 \%)$ & $11(24 \%)$ \\
\hline
\end{tabular}


FICE (mean per patient $22 \pm 5$ ). No neoplasia was found in either $\operatorname{arm}(\triangleright$ Table 2$)$.

\section{Differentiation of neoplastic and non-neoplastic lesions in targeted biopsies}

Among the 275 visible lesions analyzed in the WLE $(n=139)$ and FICE ( $n=136)$ arm, 27 (19\%), $17(12.5 \%)$ and 36 (26\%) were suspected for neoplasia by WLE, FICE-KUDO/IBD, and FICEKUDO. The number of lesions suspected for neoplasia was significantly higher using FICE-KUDO than FICE-KUDO/IBD ( $P=$ 0.004) ( $\triangleright$ Table 2).

Within all inflammatory lesions, the number of false positives was similar between FICE-KUDO (18/104) and WLE (17/ 117), but significantly less with FICE-KUDO/IBD (3/104) than with the other two methods $(P=0.001$ and $P=0.003$, respec- tively). In contrast, no differences were found between the two FICE arms and WLE in rates of false positives and negatives within hyperplasic and neoplastic lesions, respectively.

As shown in $>$ Table 3 , the sensitivity of both FICE methods was higher than that for WLE (93\% vs 64\%), but not significantly by per lesion analysis $(P=0.065)$. Nonetheless, the specificity, positive-LR and PPV of FICE-KUDO/IBD compared with WLE were $97 \%$ vs $86 \%(P=0.002), 28.3$ vs $4.5(P=0.001)$ and $76 \%$ vs $33 \%(P=0.005)$, respectively, while the negative-LR was 0.07 vs $0.42(P=0.092)$ and the NPV was $99 \%$ vs $96 \%(P=$ 0.083 ). FICE-KUDO/IBD had also higher specificity ( $97 \%$ vs 81 $\% ; P=0.001)$, positive-LR (28.3 vs $4.9 ; P=0.001)$ and PPV $(76 \%$ vs $36 \% ; P=0.006)$ than FICE-KUDO, while having similar sensitivity and NPV. Notably, NPV was above the PIVI threshold (96\% to $99 \%)$ with all three methods, without significant differences.

Table2 Rate of neoplasia according to different types of visible lesions (polypoid vs non-polypoid, suspected for or not suspected for neoplasia, flat or raised visible), and endoscopic criteria for differential diagnosis.

\begin{tabular}{|c|c|c|c|c|c|c|}
\hline & & \multirow{3}{*}{$\begin{array}{l}\text { Flat normal } \\
\text { mucosa }\end{array}$} & \multicolumn{4}{|c|}{ Visible lesions } \\
\hline & & & \multicolumn{2}{|l|}{ Polypoid } & \multicolumn{2}{|c|}{ Non-polypoid } \\
\hline & & & Suspected & Not suspected & Suspected & Not suspected \\
\hline \multirow[t]{2}{*}{ WLE } & Number of lesions & 1011 & 26 & 112 & 1 & 0 \\
\hline & Dysplasia & $0(0 \%)$ & $9(35 \%)$ & $5(4.5 \%)$ & $0(0 \%)$ & $0(0 \%)$ \\
\hline \multirow[t]{2}{*}{ FICE-KUDO } & Number of lesions & \multirow{4}{*}{$\begin{array}{l}992 \\
0(0 \%)\end{array}$} & 29 & 99 & 7 & 1 \\
\hline & Dysplasia & & $8(28 \%)$ & $1(1 \%)$ & $5(71 \%)$ & $0(0 \%)$ \\
\hline \multirow[t]{2}{*}{ FICE-KUDO/IBD } & Number of lesions & & 11 & 117 & 6 & 2 \\
\hline & Dysplasia & & $8(73 \%)$ & $1(0.9 \%)$ & $5(83 \%)$ & $0(0 \%)$ \\
\hline
\end{tabular}

WLE, white-light endoscopy; FICE, Fuji Intelligent Color Enhancement; KUDO, Kudo classification; IBD, inflammatory bowel disease.

- Table 3 Diagnostic performance of WLE versus FICE (according to the Kudo or the new classification) in the protocol of targeted sample of visible lesions (per lesion and per patient analysis).

\begin{tabular}{|c|c|c|c|c|c|c|}
\hline & \multicolumn{3}{|l|}{ Per Lesion } & \multicolumn{3}{|l|}{ Per Patient } \\
\hline & WLE & FICE-NEW & FICE-KUDO & WLE & FICE-NEW & FICE-KUDO \\
\hline Sensitivity $(95 \% \mathrm{Cl})$ & $\begin{array}{l}0.64 \\
(0.35-0.87)\end{array}$ & $\begin{array}{l}0.93 \\
(0.66-0.99)\end{array}$ & $\begin{array}{l}0.93 \\
(0.66-0.99)\end{array}$ & $\begin{array}{l}0.54 \\
(0.23-0.83)\end{array}$ & $\begin{array}{l}0.91 \\
(0.59-0.99)\end{array}$ & $\begin{array}{l}0.91 \\
(0.59-0.99)\end{array}$ \\
\hline Specificity (95\%Cl) & $\begin{array}{l}0.86 \\
(0.79-0.92)\end{array}$ & $\begin{array}{l}0.97 \\
(0.94-0.99)^{1,2}\end{array}$ & $\begin{array}{l}0.81 \\
(0.74-0.88)\end{array}$ & $\begin{array}{l}0.65 \\
(0.49-0.79)\end{array}$ & $\begin{array}{l}0.91 \\
(0.77-0.98)^{1}\end{array}$ & $\begin{array}{l}0.71 \\
(0.54-0.85)\end{array}$ \\
\hline Positive likelihood ratio $(95 \% \mathrm{Cl})$ & $\begin{array}{l}4.5 \\
(2.5-8.0)\end{array}$ & $\begin{array}{l}28.3 \\
(10.7-75.1)^{1,2}\end{array}$ & $\begin{array}{l}4.9 \\
(3.3-7.3)\end{array}$ & $\begin{array}{l}1.6 \\
(0.8-3.1)\end{array}$ & $\begin{array}{l}10.6 \\
(3.5-31.8)^{1,2}\end{array}$ & $\begin{array}{l}3.2 \\
(1.8-5.5)\end{array}$ \\
\hline Negative likelihood ratio $(95 \% \mathrm{Cl})$ & $\begin{array}{l}0.42 \\
(0.2-0.8)\end{array}$ & $\begin{array}{l}0.07 \\
(0.01-0.49)\end{array}$ & $\begin{array}{l}0.09 \\
(0.01-0.96)\end{array}$ & $\begin{array}{l}0.7 \\
(0.35-1.4)\end{array}$ & $\begin{array}{l}0.1 \\
(0.01-0.65)\end{array}$ & $\begin{array}{l}0.1 \\
(0.02-0.83)\end{array}$ \\
\hline Positive predictive value $(95 \% \mathrm{Cl})$ & $\begin{array}{l}0.33 \\
(0.22-0.47)\end{array}$ & $\begin{array}{l}0.76 \\
(0.55-0.90)^{1,2}\end{array}$ & $\begin{array}{l}0.36 \\
(0.28-0.46)\end{array}$ & $\begin{array}{l}0.29 \\
(0.17-0.44)\end{array}$ & $\begin{array}{l}0.77 \\
(0.53-0.91)^{1}\end{array}$ & $\begin{array}{l}0.50 \\
(0.36-0.64)\end{array}$ \\
\hline Negative predictive value $(95 \% \mathrm{Cl})$ & $\begin{array}{l}0.96 \\
(0.91-0.98)\end{array}$ & $\begin{array}{l}0.99 \\
(0.95-0.99)\end{array}$ & $\begin{array}{l}0.99 \\
(0.94-0.99)\end{array}$ & $\begin{array}{l}0.85 \\
(0.74-0.92)\end{array}$ & $\begin{array}{l}0.97 \\
(0.83-0.99)\end{array}$ & $\begin{array}{l}0.96 \\
(0.79-0.99)\end{array}$ \\
\hline \multicolumn{7}{|c|}{$\begin{array}{l}\text { WLE, white-light endoscopy; FICE, Fuji Intelligent Color Enhancement; NEW, new classification; KUDO, Kudo classification } \\
1 P<0.05 \text { vs WLE } \\
2 P<0.05 \text { vs FICE-KUDO }\end{array}$} \\
\hline
\end{tabular}


Similar results were obtained in the per patient analysis ( Table3). Notably, NPVs were not significantly different among the three methods but were above the PIVI threshold only with FICE, irrespective of the modified Kudo classification (96- $97 \%$ vs $85 \%)$.

\section{Discussion}

Our study is the first prospective trial evaluating FICE in realworld endoscopic surveillance for UC. Using a pragmatic study design that included multiple morphological and histological types of lesions, our study demonstrates that FICE is more accurate than standard WLE in differentiation of visible lesions only if it is used with a modified Kudo classification adapted for IBD.

The most original feature of our study was use, for the first time in a prospective study, of a modified Kudo classification, which we previously reported in a pilot study based on systematic analysis of 205 raised lesions found in 59 consecutive patients with long-standing UC [3]. In that study, accuracy of the conventional Kudo classification was unsatisfactory, but a retrospective post-hoc analysis revealed that it would have been significantly improved after the addition of further endoscopic markers: presence of a fibrin cap, significantly associated with inflammatory lesions, reclassified as unsuspected for neoplasia those lesions unclassified according to conventional Kudo or type IIIL-IV suspected neoplastic lesions, while presence of irregular vessels or pits heterogeneity reclassified a lesion as suspicious for neoplasia despite type-I or type-II Kudo pit patterns.

The need to develop new classifications, specific for surveillance in IBD, is not new, although no specific conclusions have been drawn. Previous studies using dye-based chromoendoscopy $[4,19,20]$ and NBI $[21,22]$ showed that the conventional Kudo classification of mucosal pit-patterns used in the general population is not accurate enough to differentiate between neoplastic and non-neoplastic lesions in IBD. More recently, in the FACILE classification developed for i-SCAN in IBD [23], the Kudo criteria were not integrated in the final multivariate analysis of endoscopic criteria of neoplasia to avoid uncertainty in the absence of magnified endoscopy and in the presence of regenerative changes of the mucosa. However, the Kudo classification is often used by Eastern endoscopists and by many European endoscopists who translate their usual approach for CRC screening in the general population to patients with IBD.

The debate on accuracy and reproducibility of endoscopic markers of neoplasia has an important clinical and scientific implication, because variability in diagnostic performance of various endoscopic methods of surveillance in IBD (with positive results reported mostly with dye-based chromoendoscopy rather than virtual chromoendoscopy) can be explained, at least in part, by limitations in the classification system used to identify lesions as suspicious or not suspicious for neoplasia, rather than by the imaging technology itself. Notably, if our study had used the conventional Kudo classification, it would have been a further failure for virtual chromoendoscopy in $\mathrm{IBD}$, as was the case in most previous trials with non-magnified
$\mathrm{NBI}[8,21]$ : the accuracy of FICE with conventional Kudo was in fact similar to WLE.

The need to critically reanalyze the role of chromoendoscopy based on more specific rules in IBD depends on the different epidemiology of endoscopically visible lesions compared to the screening programs for CRC in the general population.

First, the most frequent visible lesions in IBD are inflammatory. This was confirmed by our study, in which approximately $80 \%$ of lesions found in our consecutive patients were inflammatory. Second, inflammatory lesions are more likely to be false positives for neoplasia, according to conventional criteria developed for WLE or the Kudo classification. Therefore, their prevalence in the sample of any study on chromoendoscopy in IBD can strongly influence the accuracy of the tested diagnostic methods. This was clearly shown in our study, in which WLE and FICE-KUDO had an overall similar rate of false positives, while FICE-KUDO/IBD was better than the other two methods only in the high number of inflammatory lesions.

In our opinion, inclusion of inflammatory lesions is a crucial point for any study testing the accuracy of advanced endoscopy in IBD. Unfortunately, one limitation of previous studies on surveillance endoscopy was the frequent exclusion (or partial inclusion) of inflammatory lesions. That could have led not only to simplification of the real endoscopic scenario in IBD but also to overestimation of the accuracy of endoscopy, because inflammatory lesions are often difficult to differentiate from neoplastic lesions, based on a bias of pit patterns related to the effect of the inflammatory infiltrate of the mucosa [3,24].

Thanks to our inclusion criteria, this study suggests a welldefined role for FICE in differential diagnosis of any type of lesion in UC (polypoid or non-polypoid), in that it correctly characterized $93 \%$ of neoplasms and $97 \%$ of non-neoplastic lesions. With higher specificity than WLE and the best combination of positive and negative likelihood ratios (with a positive $L R>10$, a negative $L R<0.1$ and $a+L R /-L R$ ratio around 400 ), FICEKUDO/IBD appears to be the most accurate diagnostic method in our sample.

In our study, the greatest contribution of FICE-KUDO/IBD was its specificity (91\%), which was competitive with the variable rates reported in IBD with dye-based chromoendoscopy ( $57 \%$ to $97 \%)[4,19,25,26]$ and quite higher than the range reported with not-magnified NBI (66\%-81\%) [22, 27, 28]. From a clinical point of view, such a rate can appear high enough to support endoscopists in assessment of multiple lesions throughout the colon in patients with IBD. Moreover, the very high (97\% to $99 \%$ ) NPV of FICE-KUDO/IBD in both per-patient and per-lesion analysis addressed the $90 \%$ threshold established by the ASGE for accuracy required for a "diagnose and leave" strategy for more frequent non-neoplastic lesions, although that strategy requires further study specifically in neoplasia associated with IBD [18].

The high sensitivity ( $91 \%$ ) reported with FICE-KUDO/IBD in our study is higher than the pooled sensitivity (83\%) shown in patients with IBD in a recent meta-analysis on dye-chromoendoscopy [25], and clearly higher than the rate (75\% to $76 \%$ ) reported with non-magnified NBI in IBD $[22,27]$, although direct comparison is not possible due to different inclusion criteria 
and study design. Nevertheless, the high sensitivity did not reach statistical significance as compared with WLE. We believe that may be due to the experience of the endoscopists at our IBD tertiary center even when using WLE. On the other hand, the absolute high sensitivity of FICE may be due to the different resolutions of the two imaging systems. High-definition plus magnification imaging used with FICE can better analyze the mucosal surface than can the standard definition used in our WLE arm, as previously described in patients who did not have IBD [29], but less clearly in patients with IBD [9,29-32]. Certainly, further studies are needed in independent cohorts comparing high-definition endoscopy with or without FICE-chromoendoscopy.

Our study was not conceived to analyze the detection rate for the two endoscopic imaging methods because of its parallel design. Rather, it was developed to study the accuracy of differential diagnosis in two comparable populations. Therefore, the rate of neoplastic lesions had to be similar at the end of the non-randomized, prospective, patient allocation and the detection rate could not be an independent outcome. However, within visible lesions, our study seems to suggest that FICE can more easily detect non-polypoid lesions because a higher number of such lesions, among all consecutive spontaneous visible lesions included, were found compared with the WLE group. Recently, non-polypoid morphology was found to be an independent endoscopic marker of neoplasia in IBD in a study using high-definition endoscopy with i-SCAN [23]. Globally, our data suggest higher accuracy of FICE than WLE for lesions that are more difficult to visualize and clinically important.

Notably, this also was recently underscored for dye-based chromoendoscopy in a meta-analysis comparing it with WLE. Dye-based chromoendoscopy was superior to WLE in detecting non-polypoid (but not polypoid) dysplastic lesions [33]. Conversely, no additional role was found for random biopsies when systematic analysis of the entire colorectal mucosa was supported by powered endoscopy. Despite the significant number random biopsies done in our study, and their related costs and time, no false negatives for neoplasia were found.

Our study has several limitations. First, it was performed in a single center using a modified Kudo classification, which has never been validated before outside our IBD center. Therefore, a multicenter study on intraobserver and interobserver agreement is ongoing. Second, the study was not randomized and the timely patient allocation was not homogeneous. The parallel study design may be associated with more potential bias in low sample sizes compared with crossover studies in which testing is done on the same lesions with both techniques. In our opinion, however, it would be impossible to perform randomized crossover studies because of the many clinical, endoscopic, and technical variables that would have to be reconciled in this complex clinical setting. On the other hand, in our study, all efforts were made to ensure that the case-control groups were comparable, including comparable lesions from a quantitative and qualitative point of view.

\section{Conclusion}

In conclusion, our study suggests that a protocol based only on targeted biopsies of suspected neoplastic lesions appears to be the best approach we can perform with a virtual chromoendoscopy technique like FICE in differentiation of polypoid and nonpolypoid lesions in UC, if the adapted IBD specific classification, such as our modified Kudo classification, is used. Further studies in independent cohorts are required to show the reproducibility of such a classification and to confirm its diagnostic yield when used with other chromoendoscopy techniques and/or high-definition instruments.

\section{Competing interests}

The authors declare that they have no conflict of interest.

\section{References}

[1] Van Assche G, Dignass A, Bokemeyer B et al. Second European evidence-based consensus on the diagnosis and management of ulcerative colitis. Part 3: Special situations. J Crohn Colitis 2013; 7: 1-33

[2] Lambert R, Kudo SE, Vieth M et al. Pragmatic classification of superficial neoplastic colorectal lesions. Gastrointest Endosc 2009; 70: 1182-1199

[3] Cassinotti A, Buffoli F, Fociani P et al. Virtual chromoendoscopy with FICE in the classification of polypoid and non polypoid raised lesions in ulcerative colitis. J Clin Gastroenterol 2019; 53: 269-276

[4] Kiesslich R, Fritsch J, Holtmann M et al. Methylene blue-aided chromoendoscopy for the detection of intraepithelial neoplasia and colon cancer in ulcerative colitis. Gastroenterology 2003; 124: 880-888

[5] Subramanian V, Mannath J, Ragunath K et al. Meta-analysis: the diagnostic yield of chromoendoscopy for detecting dysplasia in patients with colonic inflammatory bowel disease. Aliment Pharmacol Ther 2011; 33: 304-312

[6] Nagata S, Tanaka S, Haruma K et al. Pit pattern diagnosis of early colorectal carcinoma by magnifying colonoscopy: clinical and histological implications. Int J Oncol 2000; 16: 927-934

[7] Laine L, Kaltenbach T, Barkun A et al. SCENIC international consensus statement on surveillance and management of dysplasia in inflammatory bowel disease. Gastrointest Endosc 2015; 81: 489-501

[8] Bisschops R, East J, Kaminski M et al. Advanced imaging for detection and differentitatio of colorectal neoplasia: European Society of Gastrointestinal Endoscopy (ESGE) guideline - Udate 2019. Endoscopy 2019; 51: 1155-1179

[9] lacucci M, Kaplan GG, Panaccione R et al. A randomized trial comparing high definition colonoscopy alone with high definition dye spraying and electronic virtual chromoendoscopy for detection of colonic neoplastic lesions during IBD surveillance colonoscopy. Am J Gastroenterol 2018; 113: 225-234

[10] Parra-Blanco A, Jimenez A, Rembacken B et al. Validation of Fujinon intelligent chromoendoscopy with high definition endoscopes in colonoscopy. World J Gastroenterol 2009; 15: 5266-5273

[11] Ueno Y, Tanaka S, Chayama K. Non-polypoid colorectal neoplasms in ulcerative colitis. Gastrointest Endosc Clin N Am 2010; 20: 525-542

[12] Teixeira CR, Torresini RS, Canali $C$ et al. Endoscopic classification of the capillary-vessel pattern of colorectal lesions by spectral estimation technology and magnifying zoom imaging. Gastrointest Endosc 2009; 69: 750-755 
[13] Matsumoto T, Kudo T, Jo Y et al. Magnifying colonoscopy with narrow band imaging system for the diagnosis of dysplasia in ulcerative colitis: a pilot study. Gastrointest Endosc 2007; 66: 957-965

[14] Nishiyama S, Oka S, Tanaka S. Is it possible to discriminate between neoplastic and non neoplastic lesions in ulcerative colitis by magnifying colonoscopy? Inflamm Bowel Dis 2014; 20: 508-513

[15] Rubin P, Friedman S, Harpaz $N$ et al. Colonoscopic polypectomy in chronic colitis: conservative management after endoscopic resection of dysplastic polyps. Gastroenterology 1999; 117: 1295-1300

[16] Sussman D, Barkin J, Martin A et al. Development of advanced imaging criteria for the endoscopic identification of inflammatory polyps. Clin Transl Gastroenterol 2015; 6: e128

[17] Schlemper RJ, Riddell RH, Kato Y et al. The Vienna classification of gastrointestinal epithelial neoplasia. Gut 2000; 47: 251-255

[18] Rex DK, Kahi C, O'Brien M et al. The American Society for Gastrointestinal Endoscopy PIVI (Preservation and Incorporation of Valuable Endoscopic Innovations) on real-time endoscopic assessment of the histology of diminutive colorectal polyps. Gastrointest Endosc 2011; 73: 419-422

[19] Hlavaty T, Huorka M, Koller T et al. Colorectal cancer screening in patients with ulcerative and Crohn's colitis with use of colonoscopy, chromoendoscopy and confocal endomicroscopy. Eur J Gastroenterol Hepatol 2011; 23: 680-689

[20] Nishiyama S, Oka S, Tanaka S. Is it possible to discriminate between neoplastic and non neoplastic lesions in ulcerative colitis by magnifying colonoscopy? Inflamm Bowel Dis 2014; 20: 508-513

[21] East JE, Suzuki N, von Herbay A et al. Narrow band imaging with magnification for dysplasia detection and pit pattern assessment in ulcerative colitis surveillance: a case with multiple dysplasia associated lesions or masses. Gut 2006; 55: 1432-1435

[22] van den Broek F], Fockens P, van Eeden S et al. Endoscopic tri-modal imaging for surveillance in ulcerative colitis: randomised comparison of high-resolution endoscopy and autofluorescence imaging for neoplasia detection; and evaluation of narrow-band imaging for classification of lesions. Gut 2008; 57: 1083-1089
[23] lacucci M, McQuaid K, Gui XS et al. A Multimodal (FACILE) classification for optical diagnosis of inflammatory bowel disease associated neoplasia. Endoscopy 2019; 51: 133-141

[24] Kunihiro M, Tanaka S, Sumii M et al. Magnifying colonoscopic features of ulcerative colitis reflect histologic inflammation. Inflamm Bowel Dis 2004; 10: 737-744

[25] Wu L, Li P, Wu J et al. The diagnostic accuracy of chromoendoscopy for dysplasia in ulcerative colitis: meta-analysis of six randomized controlled trials. Colorectal Dis 2010; 14: 416-420

[26] Hurlstone DP, Sanders DS, Lobo AJ et al. Indigo carmine-assisted highmagnification chromoscopic colonoscopy for the detection and characterization of intraepithelial neoplasia in ulcerative colitis: a prospective evaluation. Endoscopy 2005; 37: 1186-1192

[27] van den Broek FJ, Fockens P, van Eeden S et al. Narrow-band imaging versus high-definition endoscopy for the diagnosis of neoplasia in ulcerative colitis. Endoscopy 2011; 43: 108-115

[28] Matsumoto T, Kudo T, Jo Y et al. Magnifying colonoscopy with narrow band imaging system for the diagnosis of dysplasia in ulcerative colitis: a pilot study. Gastrointest Endosc 2007; 66: 957-965

[29] Subramanian V, Mannath J, Hawkey C] et al. High definition colonoscopy vs. standard video endoscopy for the detection of colonic polyps: a meta-analysis. Endoscopy 2011; 43: 499-505

[30] Hata K, Watanabe T, Motoi T. Pitfalls of pit pattern diagnosis in ulcerative colitis-associated dysplasia. Gastroenterology 2004; 126 : 374-376

[31] Subramanian V, Ramappa V, Telakis E et al. Comparison of high definition with standard white light endoscopy for detection of dysplasic lesions during surveillance colonoscopy in patients with colonic inflammatory bowel disease. Inflamm Bowel Dis 2013; 19: 350-355

[32] Carballal S, Maisterra S, Lopez-Serrano A et al. Real-life chromoendoscopy for neoplasia detection and characterization in long-standing IBD. Gut 2018; 67: 70-78

[33] Wan J, Wang X, Yang ZP et al. Systematic review with eta-analysis: chromoendoscopy versus white-light endoscopy in detection of dysplasia in patients with inflammatory bowel disease. J Dig Dis 2019; 20 : 206-214 